\title{
Medication related osteonecrosis of the auditory canal
}

Vittorio Fusco ${ }^{1}$, Alberto Bedogni ${ }^{2}$, Rodolfo Mauceri ${ }^{3}$, Giuseppina Campisi ${ }^{3}$

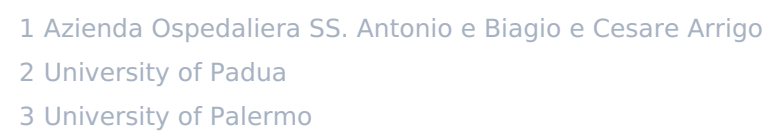

Potential competing interests: The author(s) declared that no potential competing interests exist.

Medication related osteonecrosis of the auditory canal is an adverse drug reaction described as the progressive destruction and death of bone that affects the auditory canal of patients exposed to the treatment with medications known to increase the risk of disease, in the absence of a previous radiation treatment. 